\title{
Magnetohydrodynamic Fluctuating Free Convection Flow of Second-Grade Fluid Flow in a Porous Medium
}

\author{
Farhad Ali $(\mathbb{D}$, Momin Khan, and Madeha Gohar (iD \\ Department of Mathematics, City University of Science and Information Technology, Peshawar, Khyber Pakhtunkhwa, Pakistan \\ Correspondence should be addressed to Madeha Gohar; madiha.gohar@cusit.edu.pk
}

Received 23 December 2020; Revised 16 February 2021; Accepted 24 March 2021; Published 7 April 2021

Academic Editor: Zhiguo Yan

Copyright (c) 2021 Farhad Ali et al. This is an open access article distributed under the Creative Commons Attribution License, which permits unrestricted use, distribution, and reproduction in any medium, provided the original work is properly cited.

\begin{abstract}
The article's objective is to study the magnetohydrodynamic fluctuating free convection flow of incompressible electrically conducting viscoelastic fluid in a porous medium in the presence of a pressure gradient. The flow is between two parallel plates; the lower plate is at rest, while the upper plate moves with a free stream velocity $U(t)$. A uniform magnetic field of strength $B_{0}$ is applied transversely to the fluid motion. The dimensional governing equations and physical initial and boundary conditions are nondimensionalized by inserting appropriate dimensionless variables. Furthermore, Lighthill's method is used to obtain exact analytical solutions for the velocity, temperature, and concentration distributions. Finally, the influences of inserted parameters are studied through plots and discussed physically. The numerical results are calculated and presented in tabular form for skin friction. It is worth mentioning that when the viscoelastic parameter $\alpha_{1}$ tends to zero, the obtained general solution is reduced to Newtonian fluid, which shows the validity and correctness of our obtained exact solutions.
\end{abstract}

\section{Introduction}

The understanding of flow characteristics of non-Newtonian fluid has become a subject of interest due to its widespread applications in industries and engineering, and it has been studied by Chhabra and Richardson [1]. Such interested motivations of a large number of applications have been studied in different fields such as geophysics, biology, chemistry, and petroleum industries [2]. The non-Newtonian fluids have been considered as more appropriate fluids than Newtonian fluids in industrial and technological applications. Because of particular applications of nonNewtonian fluid, it has become an increasingly applied topic of central research. These fluids fall into three classes known as integral, differential, and rate type. The differential sort of non-Newtonian fluids called viscoelastic type fluids has distinct characteristics compared to the viscous materials due to their higher-order governing equations compared to Navier-Stokes equations. Specific examples of non-Newtonian fluids are tomato sauce, polymers, and blood. The analysis of non-Newtonian fluids flow is significant in the field of engineering and oil and gas purification and filtration process. Researchers have reported on viscoelastic fluid flow applications using a porous medium [3-6].

MHD has a vital role in the field of engineering and industrial level. The magnetohydrodynamics fluid flow has great interest due to its importance in MHD generator, designing the cooling system with liquid metal, MHD pumps accelerators, and flow meter. Characteristics of magnetic field effects have significant applications in science and engineering, such as MHD pumps, geothermal energy extraction, MHD generators, and many more. A useful review of MHD theory and its applications has been found in Moreau's book [7]. MHD is the study of the magnetic properties and behavior of electrically conducting fluids. Examples of such magnetofluids include plasmas, liquid metals, salt water, and electrolytes. The word "magnetohydrodynamics" was derived from the word "magneto," meaning magnetic field, the word "hydro," meaning water, and the word "dynamics," meaning movement. The field of MHD was initiated by Hannes Alfvén [8], for which he received the Nobel Prize in Physics in 1970. The fundamental concept behind MHD is that magnetic fields can induce currents in a moving conductive fluid, which in turn 
polarizes the fluid and reciprocally changes the magnetic field itself. The set of equations that describe MHD are a combination of the Navier-Stokes equations of fluid dynamics and Maxwell's equations of electromagnetism. These differential equations have been solved simultaneously, either analytically or numerically. MHD applications have been seen in plasma, which is strongly collisional. The collisions' time scale is shorter than the other characteristic times in the system, and the particle distributions are therefore close to Maxwellian. Interest in length scales much longer than the ion skin depth and Larmor radius perpendicular to the field, long enough along the field to ignore Landau damping, and time scales much longer than the ion gyration time. The importance of significant contributions of MHD in the boundary layer flow has increased among the researchers day by day. Many researchers have examined the MHD flow through a porous medium. The applications of viscoelastic fluid MHD flow have been studied in [9-13]. Makinde and Mhone [11] have discussed the unsteady MHD flow with slip boundary conditions. In another work, Makinde and Osalusi [14] have written a brief discussion on the MHD steady flow with slip and porous boundaries with uniform suction velocity. Makinde and Aziz [15] have investigated the MHD flow considering a porous medium. Barik et al. [16] have debated on the influence of MHD oscillatory flow through short effect. Sivaraj and Kumar [17] have investigated the impact of chemical reactions with a varying concentration on MHD flow. Singh [18] has derived an exact solution for MHD flow in a channel taking porous medium. That work is comprehensively discussed by Mehmood and Ali [19] considering slip condition. The perturbation technique has been used by Kumar et al. [20] to solve the similar problem of Mehmood and Ali [19] who considered the influence of slip boundary conditions. Farhad et al. [21] investigated unsteady magnetohydrodynamic oscillatory flow in a porous channel with heat and mass transfer. Baoku et al. [22] have discussed the effect of thermal radiation on MHD flow in a porous medium. Motsa and Shateyi [23] discussed numerically mixed convection magnetohydrodynamic flow under the effect of ohmic heating. Bakr [24] and Prakash et al. [25] have discussed the impact of unsteady MHD mixed convective flow over a moving porous plate. The nonlinear MHD flow with heat and mass transfer of an incompressible, viscoelastic fluid on a stretching surface and stratification effects have been investigated by Kandasamy et al. [26]. Ishak et al. [27] examined two-dimensional MHD stagnation point flow towards stretching sheet with variable surface temperature. Akbar et al. [28] have analyzed the MHD flow of viscoelastic fluids taking a porous medium. The study of MHD free convection flow of viscoelastic fluid through a porous wedge has been studied by Rashidi et al. [29]. Jang and Lee [30] investigated the micro pump study, where pumping mechanism was based on the MHD principles and electrically conducting fluids in electric and magnetic fields.

The sum of all shear stresses of the second order is called second-grade fluids. Non-Newtonian fluids can be further categorized into three types on the shear bases: differential- type fluid, rate-type fluid, and integral-type fluid. The second-grade fluid is a subtype of the differential type of fluids. Erdogan and Irmak [31] have studied the transitory flow of second-grade fluid and reported that pressure is the cause of the fluid's spontaneous velocity. An analytical solution for the velocity of second-grade fluid has been derived by Fetecau and zierep [32]. Erdogan and Irmak [33] discussed an analytical solution for the governing equation of velocity of a second-grade fluid in three-dimensional flows. Nazar et al. [34] obtained a few closed-form results using the Laplace transform method for oscillatory second-grade fluid flow. This work was further extended by Ali et al. [35] from nonporous hydrodynamic to porous hydromagnetic secondgrade fluid flow to obtain closed-form solutions using the same technique. Unsteady MHD flow of second-grade fluid over an oscillating vertical plate in a porous medium with heat and mass transfer has been investigated by Farhad et al. [36]. The homotopy analysis method (HAM) has been used by Hayat et al. [37] for viscoelastic fluid flow to the derived analytical solution for velocity and temperature profile. Anwar et al. [38] have discussed the laminar flow of second grade with a horizontal cylinder. The steady magnetohydrodynamic flow of viscoelastic fluid is investigated by Eldabe et al. [39]. Mustafa [40] has considered a transverse magnetic field through a porous medium of second-grade fluid in a tube. Shah and Khan [41] have discussed the convective flow of second-grade fluid to obtain exact solutions for heat and velocity by Laplace transforms technique.

The velocity of the fluid above the boundary layer or free stream velocity is defined as the speed at which the air is infinitely far from the aircraft moving when the object is kept as referential called free stream velocity. Free stream velocity is always greater than the boundary layer velocity because free stream velocity starts from the upper boundary of the fluid's boundary layer velocity. In (1954), Lighthill has studied the laminar boundary layer, time-dependent free stream oscillation. An unsteady mixed convection flow over a moving vertical plate in a vertical free stream with combined effects of boundary force and thermal diffusion has been studied by Patil et al. [42]. A uniform free stream with a fixed or moving surface has been investigated by Ishak et al. [43]. The nonsimilar boundary layer flow of viscous fluid over a moving surface in a rotating fluid with free stream velocity has been explained by Takhar et al. [44]. The effect of horizontal oscillations of the free stream velocity on pitching airfoil oscillations using computational fluid dynamics has been studied by Gharali and Johnson [45]. Alassar et al. [46] investigated the viscous flow over a sphere with fluctuations in the free stream velocity. The effect of large temperature with the determination of skin friction for a laminar boundary layer flow with variable free stream velocity has been examined by Levy et al. [47]. Kelly [48] has studied the steady viscous flow near a stagnation point following a change free stream velocity with a finite instant of time in two dimensions. Mei and Adrian [49] have investigated unsteady flow over a stationary with small fluctuation in the free stream velocity at a finite Reynolds number. Rott et al. [50] have presented a complete solution of the laminar 
boundary layer flow of small fluctuations of the free stream velocity.

Bearing in mind the above literature review, we found a gap that no one has considered the free stream fluctuating velocity on the boundary in their studies. Therefore, to fill this gap, we considered in the present work free convective, unsteady MHD flow of incompressible, electrically conducting, second-grade fluid flow, flowing through a porous medium. Furthermore, free stream velocity and pressure gradient are taken into account. Lighthill's [51] method has been applied to obtain analytical solutions for the velocity, temperature, and concentration distributions. The effect of various embedded parameters on the skin friction is shown in tabular form. The graphs are sketched on the velocity, temperature, and concentration profiles to show the effect of several embedded parameters.

\section{Mathematical Formulation}

An incompressible, viscoelastic, second-grade fluid flow through a porous medium has been considered in the present work. The fluid is taken to be electrically conducting, and the magnetic field is transversely applied to the flow. As the magnetic Reynolds number is smaller, we are neglecting the induced magnetic field's influence. The fluid velocity in the boundary layer $U$ is the free stream velocity away from the boundary layer. We assume that the medium's lower boundary is at constant temperature $T_{0}$ and concentration, $C_{0}$, while the ambient fluid has temperature $T_{w}$ and concentration level $C_{w}$ uniformly. Geometry of the flow is shown in Figure 1.

The fluid flow is along the $x$-axis, and the $y$-axis is normal to the flow direction. Three main equations govern the given flow regime: one is the momentum equation, the second is the heat equation, and the third one is concentration distribution, such as [21]

$$
\begin{aligned}
\rho \frac{\partial u(y, t)}{\partial t}= & -\frac{\partial p}{\partial x}+\mu \frac{\partial^{2} u(y, t)}{\partial y^{2}}+\alpha_{1} \frac{\partial}{\partial t}\left(\frac{\partial^{2} u(y, t)}{\partial y^{2}}\right)+g \beta_{T}\left(T(y, t)-T(y, t)_{0}\right) \\
& +g \beta_{C}\left(C(y, t)-C_{0}(y, t)\right)-\sigma B_{0} u(y, t)-\frac{\phi_{1}}{k_{1}}\left(1+\frac{\alpha_{1} \partial}{\mu \partial t}\right) u(y, t), \\
\rho C_{p} \frac{\partial T(y, t)}{\partial t}= & k \frac{\partial^{2} T(y, t)}{\partial y^{2}}-\frac{\partial q_{r}}{\partial y}, \\
\frac{\partial C(y, t)}{\partial t}= & D \frac{\partial^{2} C(y, t)}{\partial y^{2}}+\left(\frac{D K_{t}}{T_{m}}\right) \frac{\partial^{2} T(y, t)}{\partial y^{2}} .
\end{aligned}
$$

Initial and boundary conditions are given as

$$
\left.\begin{array}{ll}
u(0, t)=0 & u(1, t)=1+\varepsilon e^{i \omega t} \\
T(0, t)=T_{0} & T(1, t)=T_{w} \\
C(0, t)=C_{0} & C(1, t)=C_{w} .
\end{array}\right\}
$$

The velocity, heat, and concentration equations show density, dynamic viscosity, second-grade parameter, electrical conductivity, thermal expansion coefficient, concentration expansion coefficient, gravitational acceleration and heat capacity, porosity, and permeability parameters, respectively. $D$ shows the mass diffusivity, $k$ represents thermal conductivity of the fluid, $q_{r}$ is the radiative heat flux, "a" shows the width of the channel, $K_{T}$ is the thermal diffusion ratio, and $\omega$ is the frequency of oscillation. The temperature of the plates is $T_{w}$ and $T_{0}$ which produces radiation heat transfer. The radiation flux of heat is assumed as

$$
-\frac{\partial q_{r}}{\partial y}=4 \alpha_{0}^{2}\left(T-T_{0}\right)
$$

where $\alpha_{0}$ is the radiation absorption coefficient.

For dimensional analysis, the dimensionless variables are

$$
\begin{aligned}
u^{*} & =\frac{u}{U_{o}}, \\
y^{*} & =\frac{y}{a}, \\
t^{*} & =\frac{U_{0}}{a} t, \\
\theta & =\frac{T-T_{0}}{T_{w}-T_{0}}, \\
\phi & =\frac{C-C_{0}}{C_{w}-C_{0}} .
\end{aligned}
$$

For the free stream velocity, we convert the velocity of the fluid $u(y, t)$ with free stream velocity $U(t)$ all functions being considered as zero except $U(t)$ and, thus, we get the following equation:

$$
-\frac{1}{\rho} \frac{\partial p}{\partial x}=\frac{\partial U}{\partial t}+\sigma B_{0}^{2} U+\frac{\mu \phi_{1}}{\rho k_{1}}\left(1+\frac{\alpha_{1} \partial}{\mu \partial t}\right) U .
$$

Putting equation (7) in equation (1), we get 


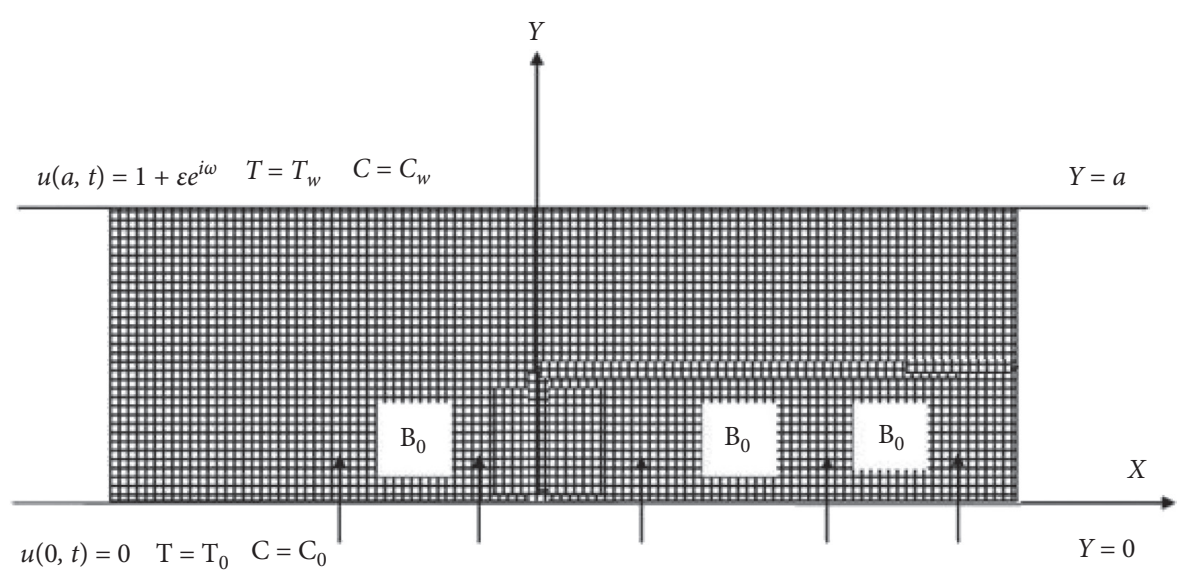

Figure 1: Geometry of the flow.

$$
\begin{aligned}
\frac{\partial u(y, t)}{\partial t}= & \frac{\partial U(t)}{\partial t}+\frac{\sigma}{\rho} B_{0}^{2} U(t)+\frac{\mu \phi_{1}}{\rho k_{1}}\left(1+\frac{\alpha_{1} \partial}{\mu \partial t}\right) U(t)+\mu \frac{\partial^{2} u(y, t)}{\rho \partial y^{2}}+\alpha_{1} \frac{\partial}{\rho \partial t}\left(\frac{\partial^{2} u(y, t)}{\partial y^{2}}\right) \\
& +\frac{g}{\rho} \beta_{T}\left(T-T_{0}\right)+\frac{g}{\rho} \beta_{C}\left(C-C_{0}\right)-\frac{\sigma}{\rho} B_{0} u(y, t)-\frac{\phi_{1}}{\rho k_{1}}\left(1+\frac{\alpha_{1} \partial}{\mu \partial t}\right) u(y, t) .
\end{aligned}
$$

After rearranging the terms, we have

$$
\begin{aligned}
\frac{\partial u(y, t)}{\partial t}= & \frac{\partial U(t)}{\partial t}+\mu \frac{\partial^{2} u(y, t)}{\rho \partial y^{2}}+\alpha_{1} \frac{\partial}{\rho \partial t}\left(\frac{\partial^{2} u(y, t)}{\partial y^{2}}\right)+\frac{g}{\rho} \beta_{T}\left(T-T_{0}\right)+\frac{g}{\rho} \beta_{C}\left(C-C_{0}\right) \\
& -\frac{\sigma}{\rho} B_{0} u(y, t)+\frac{\sigma}{\rho} B_{0}^{2} U(t)-\frac{\phi_{1}}{\rho k_{1}}\left(1+\frac{\alpha_{1} \partial}{\mu \partial t}\right) u(y, t)+\frac{\mu \phi_{1}}{\rho k_{1}}\left(1+\frac{\alpha_{1} \partial}{\mu \partial t}\right) U(t) .
\end{aligned}
$$

Taking some standard terms and then arranging, we get

$$
\begin{gathered}
\frac{\partial u(y, t)}{\partial t}=\frac{\partial U(t)}{\partial t}+\mu \frac{\partial^{2} u(y, t)}{\rho \partial y^{2}}+\alpha_{1} \frac{\partial}{\rho \partial t}\left(\frac{\partial^{2} u(y, t)}{\partial y^{2}}\right)+\frac{g}{\rho} \beta_{T}\left(T-T_{0}\right) \\
+\frac{g}{\rho} \beta_{C}\left(C-C_{0}\right)-\frac{\sigma}{\rho} B_{0}^{2}(u(y, t)-U(t))-\frac{\mu \phi_{1}}{\rho k_{1}}\left(1+\frac{\alpha_{1} \partial}{\mu \partial t}\right)(u(y, t)-U(t)), \\
\text { ndary layer velocity and } U \text { is used for the } \\
\begin{array}{cc}
a_{1} \frac{\partial u}{\partial t}-a_{1} \frac{\mathrm{d} U}{\mathrm{~d} y}-\frac{\partial^{2} u}{\partial y^{2}}-\alpha \frac{\partial}{\partial t}\left(\frac{\partial^{2} u}{\partial y^{2}}\right)+H u \\
\text { sionalization, equations (2), (3), and (10) } & -H U-\operatorname{Gr} \theta-\operatorname{Gm} \phi=0 .
\end{array}
\end{gathered}
$$

where $u$ shows boundary layer velocity and $U$ is used for the free stream velocity.

After nondimensionalization, equations (2), (3), and (10) become

$$
\begin{gathered}
\operatorname{Pe} \frac{\partial \theta}{\partial t}=\frac{\partial^{2} \theta}{\partial y^{2}}+N^{2} \theta \\
\frac{1}{\mathrm{Sc}}\left(\frac{\partial \phi}{\partial t}\right)=\frac{\partial^{2} \phi}{\partial y^{2}}+\operatorname{Sr} \frac{\partial^{2} \theta}{\partial y^{2}}
\end{gathered}
$$

Initial and boundary conditions are

$$
\left.\begin{array}{lll}
u(0, t)=0 & u(1, t)=1+\varepsilon e^{i \omega t} & t>0, \\
\theta(0, t)=1 & \theta(1, t)=0 & t>0, \\
\phi(0, t)=1 & \phi(1, t)=0 & t>0 .
\end{array}\right\}
$$


After nondimensionalization, we get some nondimensional parameters, such as

$$
\begin{aligned}
& \operatorname{Re}=\frac{\rho a U_{0}}{\mu}, \\
& \alpha=\frac{\alpha_{1} U_{0}}{\mu a}, \\
& M=\frac{\sigma B_{0}^{2} a^{2}}{\mu}, \\
& \frac{1}{k}=\frac{a^{2}}{k_{1}}, \\
& \mathrm{Gr}=g \beta_{t} a^{2} \frac{\left(T_{w}-T_{0}\right)}{\mu U_{0}}, \\
& \mathrm{Gm}=g \beta_{c} a^{2} \frac{\left(C_{w}-C_{0}\right)}{\mu U_{0}}, \\
& \mathrm{Sc}=\frac{D}{a U_{0}}, \\
& \mathrm{Sr}=\frac{K_{t}\left(T_{w}-T_{0}\right)}{T_{m}\left(C_{w}-C_{0}\right)}, \\
& \mathrm{Pe}=\frac{\rho C_{p} a U_{0}}{k}, \\
& a_{1}=\operatorname{Re}+\frac{\alpha}{k}, \\
& H=M+\frac{1}{k}, \\
& N^{2}=\frac{4 \alpha_{0}^{2} a^{2}}{k},
\end{aligned}
$$

where dimensionless viscoelastic parameter $\alpha$, the magnetic and permeability parameters $M, K$, and $\mathrm{Re}, N, \mathrm{Pe}, \mathrm{Gr}, \mathrm{Gm}, \mathrm{Sc}$, and Sr are Reynolds number, radiation, Peclet, thermal Grashof, and mass Grashof parameters, as well as Schmidt and Soret numbers, respectively.

\section{Solution of the Model}

We are solving equations (11)-(13) by Lighthill's method, and equations (16)-(18) are the assumed solutions:

$$
\begin{aligned}
& u(y, t)=u_{0}(y)+u_{1}(y) \varepsilon e^{i \omega t}+O\left(\varepsilon^{2}\right), \\
& \theta(y, t)=\theta_{0}(y)+\theta_{1}(y) \varepsilon e^{i \omega t}+O\left(\varepsilon^{2}\right),
\end{aligned}
$$

$$
\phi(y, t)=\phi_{0}(y)+\phi_{1}(y) \varepsilon e^{i \omega t}+O\left(\varepsilon^{2}\right) .
$$

By using the assumed solution and solving equations (11)-(13), we get

$$
\mathrm{Pe} \frac{\partial \theta}{\partial t}=\frac{\partial^{2} \theta}{\partial y^{2}}+N^{2} \theta .
$$

The nonharmonic part and boundary conditions for the heat equation are

$$
\begin{aligned}
\frac{\partial^{2} \theta_{0}(y)}{\partial y^{2}}+N^{2} \theta_{0}(y) & =0, \\
\theta_{0}(0) & =1, \\
\theta_{0}(a) & =0 .
\end{aligned}
$$

Solution of the harmonic part is

$$
\theta_{0}(y)=\cos N y-a_{2} \sin N y \text {. }
$$

The harmonic part and conditions for the heat equation are

$$
\begin{aligned}
\frac{\partial^{2} \theta_{1}(y)}{\partial y^{2}}+\left(N^{2}-i \omega \mathrm{Pe}\right) \theta_{1}(y) & =0, \\
\theta_{1}(0) & =0, \\
\theta_{1}(a) & =1 .
\end{aligned}
$$

Solution of the nonharmonic part is

$$
\theta_{1}(y)=0 \text {. }
$$

Putting the values of equations (21) and (23) in the assumed solution (17), we get

$$
\begin{gathered}
\theta(y, t)=\theta_{0}(y)+\theta_{1}(y) \varepsilon e^{i \omega t}+O\left(\varepsilon^{2}\right), \\
\theta(y)=\cos N y-a_{2} \sin N y .
\end{gathered}
$$

Now, we will solve the concentration equation:

$$
\frac{1}{\mathrm{Sc}}\left(\frac{\partial \phi}{\partial t}\right)=\frac{\partial^{2} \phi}{\partial y^{2}}+\operatorname{Sr} \frac{\partial^{2} \theta}{\partial y^{2}}
$$

The nonharmonic part and boundary conditions for concentration equation are

$$
\begin{aligned}
\frac{\partial^{2} \phi_{0}(y)}{\partial y^{2}}+\operatorname{Sr} \frac{\partial^{2} \theta_{0}(y)}{\partial y^{2}} & =0, \\
\phi_{0}(0) & =1, \\
\phi_{0}(a) & =0 .
\end{aligned}
$$

Solution of the nonharmonic part is

$$
\phi_{0}(y)=a_{4}+a_{3} y-\operatorname{Sr}\left(\cos N y-a_{2} \sin N y\right)
$$


The harmonic part and conditions for concentration equation are

$$
\begin{aligned}
\frac{\partial^{2} \phi_{1}(y)}{\partial y^{2}}-\frac{i \omega}{S c} \phi_{1}(y) & =-\operatorname{Sr} \frac{\partial^{2} \phi_{1}(y)}{\partial y^{2}}, \\
\phi_{1}(0) & =0 \\
\phi_{1}(a) & =0 .
\end{aligned}
$$

Solution of the harmonic part is

$$
\phi_{1}(y)=0 .
$$

Putting equations (28) and (30) in the assumed solution (18), we get

$$
\begin{aligned}
\phi(y, t) & =\phi_{0}(y)+\phi_{1}(y) \varepsilon e^{i \omega t}+O\left(\varepsilon^{2}\right), \\
\phi(y) & =a_{4}+a_{3} y-\operatorname{Sr}\left(\cos N y-a_{2} \sin N y\right) .
\end{aligned}
$$

Now solve the momentum equation:

$$
\begin{gathered}
a_{1} \frac{\partial u}{\partial t}-a_{1} \frac{\mathrm{d} U}{\mathrm{~d} y}-\frac{\partial^{2} u}{\partial y^{2}}-\alpha \frac{\partial}{\partial t}\left(\frac{\partial^{2} u}{\partial y^{2}}\right)+H u \\
-H U-\operatorname{Gr} \theta-\operatorname{Gm} \phi=0 .
\end{gathered}
$$

Putting the values of equations (25) and (32) in (33), we have

$$
\begin{aligned}
& a_{1} \frac{\partial u}{\partial t}-a_{1} \frac{d U}{d y}-\frac{\partial^{2} u}{\partial y^{2}}-\alpha \frac{\partial}{\partial t}\left(\frac{\partial^{2} u}{\partial y^{2}}\right)+H u-H U \\
& \quad-\operatorname{Gr}\left(\cos N y-a_{2} \sin N y\right) \\
& \quad-\operatorname{Gm}\left(a_{4}+a_{3} y-\operatorname{Sr}\left(\cos N y-a_{2} \sin N y\right)\right)=0 .
\end{aligned}
$$

The nonharmonic part and its conditions are

$$
\begin{aligned}
\frac{\partial^{2} u_{0}(y)}{\partial y^{2}}-H u_{0}(y)= & -\left(H+\mathrm{Gm} a_{4}\right)-(\mathrm{Gr}-\mathrm{SrGm}) \\
& \cdot\left(\cos N y-a_{2} \sin N y\right)-a_{3} \mathrm{Gm} y, \\
u_{0}(0)= & 0, \\
u_{0}(a)= & 1 .
\end{aligned}
$$

The solution of nonharmonic part is

$$
u_{0}(y)=C_{1} \cosh \sqrt{H} y+C_{2} \sinh \sqrt{H} y+\left(\frac{H+\mathrm{Gm}\left(a_{4}+a_{3} y\right)}{H}\right)+\left(\frac{\mathrm{Gr}-\mathrm{SrGm}}{H+N^{2}}\right)\left(\cos N y-a_{2} \sin N y\right) .
$$

The harmonic part and its conditions are

$$
\begin{aligned}
\frac{\partial^{2} u_{1}(y)}{\partial y^{2}}-m_{1} u_{1}(y) & =-m_{1}, \\
u_{1}(0) & =0, \\
u_{1}(a) & =1 .
\end{aligned}
$$

Solution of the harmonic part of the momentum equation is

$$
u_{1}(y)=\left(-\cosh \sqrt{m_{1}} y+a_{5} \sinh \sqrt{m_{1}} y+1\right) .
$$

Assume solution for the momentum equation:

$$
\begin{aligned}
u(y, t)= & u_{0}(y)+u_{1}(y) \varepsilon e^{i \omega t}+O\left(\varepsilon^{2}\right), \\
u(y, t)= & C_{1} \cosh \sqrt{H} y+C_{2} \sinh \sqrt{H} y+\left(\frac{H+\mathrm{Gm}\left(a_{4}+a_{3} y\right)}{H}\right) \\
& +\left(\frac{\mathrm{Gr}-\mathrm{SrGm}}{H+N^{2}}\right)\left(\cos N y-a_{2} \sin N y\right)+\varepsilon e^{i \omega t}\left(-\cosh \sqrt{m_{1}} y+a_{5} \sinh \sqrt{m_{1}} y+1\right),
\end{aligned}
$$

where $m_{1}$ is a constant:

$$
m_{1}=\frac{H+i \omega a}{1+i \omega \alpha} .
$$

We have solved heat, concentration, and momentum equations and obtained the following results: 


$$
\begin{gathered}
\theta_{1}(y)=0, \\
\phi_{1}(y)=0 . \\
\theta_{0}(y)=\cos N y-a_{2} \sin N y,
\end{gathered}
$$

$$
\begin{aligned}
& a_{2}=\frac{\cos N}{\sin N}, \\
& a_{3}=\operatorname{Sr}\left(\cos N-a_{2} \sin N\right)-1, \\
& a_{4}=1+\operatorname{Sr},
\end{aligned}
$$$$
C_{1}=-\left(\frac{H+\mathrm{Gm}\left(a_{4}+a_{3}\right)}{H}\right)-\left(\frac{\mathrm{Gr}-\mathrm{SrGm}}{H+N^{2}}\right),
$$$$
C_{2}=\frac{1}{\sinh \sqrt{H}}\left(1-C_{1} \cosh \sqrt{H}-\left(\frac{H+G m\left(a_{4}+a_{3}\right)}{H}\right)-\left(\frac{G r-\operatorname{SrGm}}{H+N^{2}}\right)\left(\cos N-a_{2} \sin N\right)\right),
$$$$
a_{5}=\frac{\cosh \sqrt{m_{1}}}{\sinh \sqrt{m_{1}}} .
$$

$$
\phi_{0}(y)=a_{4}+a_{3} y-\operatorname{Sr}\left(\cos N y-a_{2} \sin N y\right) .
$$

Using equations (43) and (44) in equation (40), we get

Now putting the values $\theta_{0}(y), \theta_{1}(y)$ and $\phi_{0}(y), \phi_{1}(y)$ in equations (26) and (31), we can obtain the following results:

$$
\begin{aligned}
\theta(y, t)= & \cos N y-a_{2} \sin N y, \quad \text { at } y=0, \\
\phi(y, t)= & a_{4}+a_{3} y-\operatorname{Sr}\left(\cos N y-a_{2} \sin N y\right), \\
u(y, t)= & C_{1} \cosh \sqrt{H} y+C_{2} \sinh \sqrt{H} y+\left(\frac{H+\mathrm{Gm}\left(a_{4}+a_{3} y\right)}{H}\right) \\
& +\left(\frac{\mathrm{Gr}-\operatorname{SrGm}}{H+N^{2}}\right)\left(\cos N y-a_{2} \sin N y\right)+\varepsilon e^{i \omega t}\left(-\cosh \sqrt{m_{1}} y+a_{5} \sinh \sqrt{m_{1}} y+1\right) .
\end{aligned}
$$

Furthermore, it has been noticed from equation (32) that the solution can be reduced into a Newtonian fluid by taking $\alpha=0$, which shows the validity of the problem.

\section{Graphical Results and Discussion}

The exact solution for free convection flow of second-grade fluid has been investigated. The governing partial differential equations and corresponding boundary conditions have been converted into ordinary differential equations and are analytically solved. Figures 2-9 are plotted to influence different physical parameters on the velocity of the fluid. Figure 10 is drawn for the parametric influence of radiation parameters for temperature profile. Figures 11 and 12 depict the effect of radiation parameter and Soret number in concentration profile. Table 1 illustrates a variation in skin friction for different parameters.
Figure 2 shows the influence of the magnetic parameter on the velocity profile; by increasing $M$, the fluid's velocity under the boundary layer decreases, which indicates the production of Lorentz force. In our case, the velocity of the fluid increases due to free steam velocity. Free stream velocity is always greater than the boundary layer velocity because the fluid's velocity increases. Physically it means that under the boundary layer velocity of the fluid decreases by increasing the magnetic parameter $M$, which causes the drag forces or frictional force, resisting the flow and reducing the velocity. The influence of Millustrates that the greater value of $M$ increases the velocity.

Figure 3 represents the velocity profile for the thermal Grashof number, increasing the velocity because of the enhancement of buoyancy force, which indicates that the thermal buoyancy force accelerates the velocity. Thermal Grashof number tells about the velocity effects of thermal 


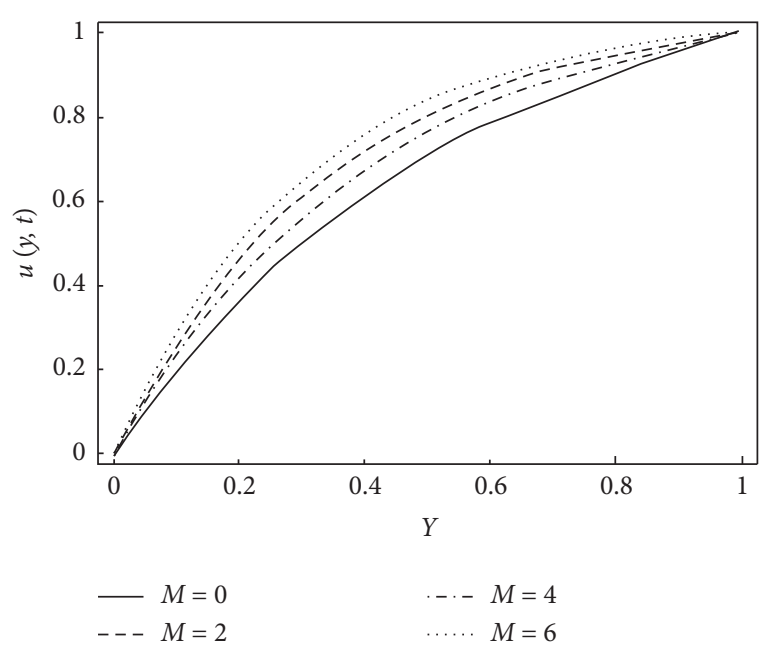

Figure 2: Velocity profile for the various values of $M$ when $\operatorname{Re}=0.2$, $\mathrm{Gr}=1.1, K=0.6, \mathrm{Gm}=1.2, N=2.2, \mathrm{Sr}=1, \alpha=0.71, \varepsilon=0.001$, and $\omega=\pi / 2$.

TABLE 1: The influence of different parameters on $\left(C_{f}\right)$ skin friction.

\begin{tabular}{lccccccccccc}
\hline & $M$ & $\mathrm{Gr}$ & $\mathrm{Gm}$ & $K$ & $N$ & $\mathrm{Sr}$ & $\alpha$ & $\varepsilon$ & $\mathrm{Re}$ & $\omega$ & $C_{f}$ \\
\hline$M=1$ & 1 & 0.5 & 1.5 & 0.02 & 2 & 2 & 0.2 & 0.1 & 1 & 0.8 & 0.5233 \\
$M=2$ & 2 & 0.5 & 1.5 & 0.02 & 2 & 2 & 0.2 & 0.1 & 1 & 0.8 & 0.5314 \\
$M=3$ & 3 & 0.5 & 1.5 & 0.02 & 2 & 2 & 0.2 & 0.1 & 1 & 0.8 & 0.5394 \\
$\mathrm{Gr}=0.5$ & 0.7 & 0.5 & 1.5 & 0.02 & 2 & 2 & 0.2 & 0.1 & 1 & 0.8 & 0.5208 \\
$\mathrm{Gr}=0.7$ & 0.7 & 0.7 & 1.5 & 0.02 & 2 & 2 & 0.2 & 0.1 & 1 & 0.8 & 0.5216 \\
$\mathrm{Gr}=0.9$ & 0.7 & 0.9 & 1.5 & 0.02 & 2 & 2 & 0.2 & 0.1 & 1 & 0.8 & 0.5221 \\
$\mathrm{Gm}=0.5$ & 0.7 & 0.5 & 0.5 & 0.02 & 2 & 2 & 0.2 & 0.1 & 1 & 0.8 & 0.5800 \\
$\mathrm{Gm}=0.6$ & 0.7 & 0.5 & 0.6 & 0.02 & 2 & 2 & 0.2 & 0.1 & 1 & 0.8 & 0.5740 \\
$\mathrm{Gm}=0.7$ & 0.7 & 0.5 & 0.7 & 0.02 & 2 & 2 & 0.2 & 0.1 & 1 & 0.8 & 0.5681 \\
$K=0.01$ & 0.7 & 0.5 & 1.5 & 0.01 & 2 & 2 & 0.2 & 0.1 & 1 & 0.8 & 0.8157 \\
$K=0.02$ & 0.7 & 0.5 & 1.5 & 0.02 & 2 & 2 & 0.2 & 0.1 & 1 & 0.8 & 0.5208 \\
$K=0.03$ & 0.7 & 0.5 & 1.5 & 0.03 & 2 & 2 & 0.2 & 0.1 & 1 & 0.8 & 0.3664 \\
$N=2$ & 0.7 & 0.5 & 1.5 & 0.02 & 2 & 2 & 0.2 & 0.1 & 1 & 0.8 & 0.5208 \\
$N=4$ & 0.7 & 0.5 & 1.5 & 0.02 & 4 & 2 & 0.2 & 0.1 & 1 & 0.8 & 0.5204 \\
$N=6$ & 0.7 & 0.5 & 1.5 & 0.02 & 6 & 2 & 0.2 & 0.1 & 1 & 0.8 & 0.5200 \\
$\mathrm{Sr}=1$ & 0.7 & 0.5 & 1.5 & 0.02 & 2 & 1 & 0.2 & 0.1 & 1 & 0.8 & 0.5504 \\
$\mathrm{Sr}=2$ & 0.7 & 0.5 & 1.5 & 0.02 & 2 & 2 & 0.2 & 0.1 & 1 & 0.8 & 0.5208 \\
$\mathrm{Sr}=3$ & 0.7 & 0.5 & 1.5 & 0.02 & 2 & 3 & 0.2 & 0.1 & 1 & 0.8 & 0.4912 \\
$\alpha=0.2$ & 0.7 & 0.5 & 1.5 & 0.02 & 2 & 2 & 0.2 & 0.1 & 1 & 0.8 & 0.5208 \\
$\alpha=0.4$ & 0.7 & 0.5 & 1.5 & 0.02 & 2 & 2 & 0.4 & 0.1 & 1 & 0.8 & 0.4769 \\
$\alpha=0.6$ & 0.7 & 0.5 & 1.5 & 0.02 & 2 & 2 & 0.6 & 0.1 & 1 & 0.8 & 0.4327 \\
$\varepsilon=0.2$ & 0.7 & 0.5 & 1.5 & 0.02 & 2 & 2 & 0.2 & 0.2 & 1 & 0.8 & 1.1304 \\
$\varepsilon=0.4$ & 0.7 & 0.5 & 1.5 & 0.02 & 2 & 2 & 0.2 & 0.4 & 1 & 0.8 & 2.3496 \\
$\varepsilon=0.6$ & 0.7 & 0.5 & 1.5 & 0.02 & 2 & 2 & 0.2 & 0.6 & 1 & 0.8 & 3.5688 \\
$\mathrm{Re}=1$ & 0.7 & 0.5 & 1.5 & 0.02 & 2 & 2 & 0.2 & 0.1 & 1 & 0.8 & 0.5208 \\
$\mathrm{Re}=2$ & 0.7 & 0.5 & 1.5 & 0.02 & 2 & 2 & 0.2 & 0.1 & 2 & 0.8 & 0.5187 \\
$\mathrm{Re}=3$ & 0.7 & 0.5 & 1.5 & 0.02 & 2 & 2 & 0.2 & 0.1 & 3 & 0.8 & 0.5166 \\
$\omega=0.1$ & 0.7 & 0.5 & 1.5 & 0.02 & 2 & 2 & 0.2 & 0.1 & 1 & 0.1 & 0.6216 \\
$\omega=0.3$ & 0.7 & 0.5 & 1.5 & 0.02 & 2 & 2 & 0.2 & 0.1 & 1 & 0.3 & 0.6086 \\
$\omega=0.5$ & 0.7 & 0.5 & 1.5 & 0.02 & 2 & 2 & 0.2 & 0.1 & 1 & 0.5 & 0.5827 \\
\hline & & & & & & & & & & &
\end{tabular}

buoyancy force to viscous force. It is observed that an increase in Gr leads to increase velocity due to enhancement in buoyancy force. Physically, Gr signifies the relative effect of the thermal buoyancy force to the viscous hydrodynamic force. Increase of Grashof number Gr means an increase of temperature gradients due to which the contribution from the buoyancy near the plate becomes significant and hence a

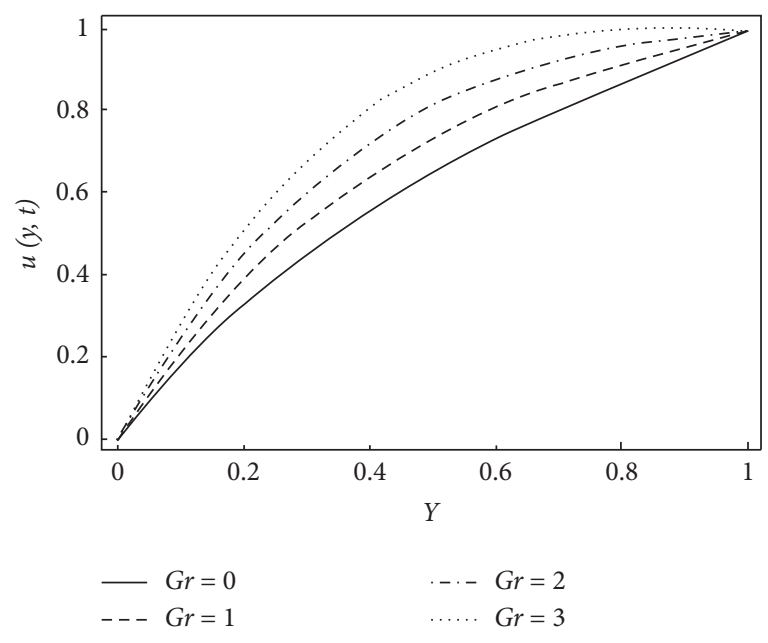

FIgURE 3: Velocity graph for the several values of $\mathrm{Gr}$ when $\mathrm{Re}=0.2$, $M=1.1, K=0.5, \mathrm{Gm}=1.1, N=2, \mathrm{Sr}=1, \alpha=0.71, \varepsilon=0.001$, and $\omega=\pi / 2$.

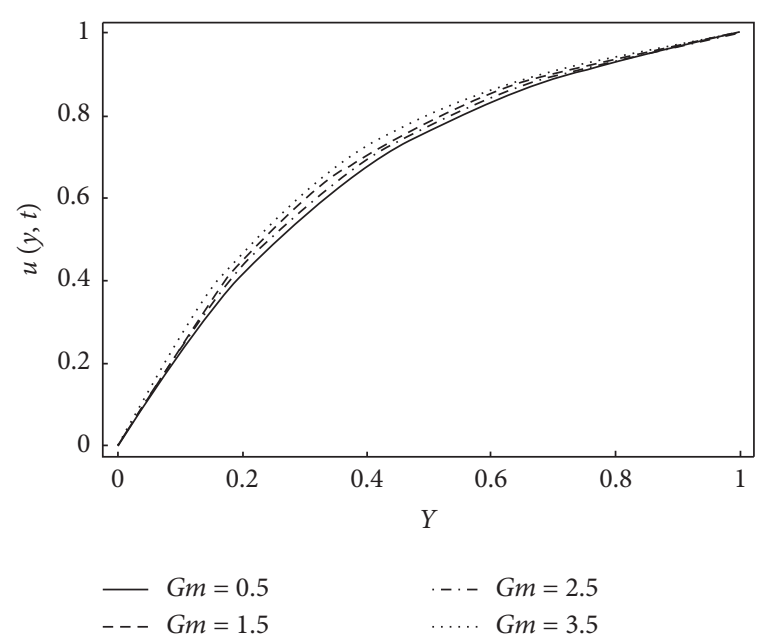

FIGURE 4: Velocity profile used for the several values of $\mathrm{Gm}$ when $\mathrm{Re}=5, \mathrm{Gr}=1.1, M=2.1, K=0.5, N=2, \mathrm{Sr}=1, \alpha=0.71, \varepsilon=0.001$, and $\omega=\pi / 2$.

short rise in the velocity near the plate is observed. $\mathrm{Gr}>0$ means heating of the fluid or cooling of the plate by natural convection. For the positive value of $\mathrm{Gr}$, heat is conducted away from the boundary into the fluid which increases the temperature and thereby enhances the buoyancy force.

Figure 4 is sketched to demonstrate the behavior of mass Grashof number on the velocity profile. It is investigating that $\mathrm{Gm}=0$ corresponds to the buoyancy force and $\mathrm{Gm}>0$ corresponds to the strong magnetic field chilling problem faced in atomic engineering connected with the chilling of the nuclear reactor. It is to be noted that a more significant number of buoyancy forces create and thus speed up the fluid flow. Boundary layer thickness becomes large along "a," where "a" is the boundary-level wideness and velocity increases $\mathrm{Gm}$.

Figure 5 illustrates the permeability parameter's impact, which shows that increasing permeability produces the drag force or resistance, decreasing the velocity. The fact is that by increasing the amount of $K$ the porous medium increases, 


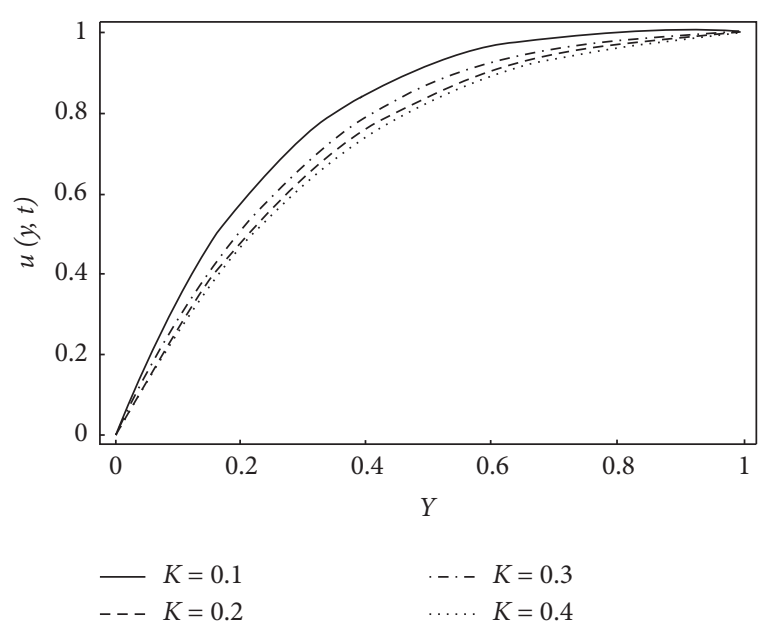

Figure 5: Velocity graph for various values of $K$ when $\operatorname{Re}=0.2$, $\mathrm{Gr}=2, M=1.1, \mathrm{Gm}=1.1, N=2, \mathrm{Sr}=1, \alpha=0.71, \varepsilon=0.001$, and $\omega=\pi / 2$.

and the opposing force becomes the cause of decreasing the velocity of the flow. Physically, it has been recorded that increasing the value of $\mathrm{K}$ increases the resistance force which decreases the velocity.

Figure 6 illustrates the influence of the viscoelastic parameter $\alpha$ on the velocity profile, which indicates that when $\alpha$ increases, velocity also increases. The area of the boundary layer increases in the center point, where $\alpha$ increases. The viscoelastic parameter $\alpha$ near the boundary $y=0$ is weaker and increases far away from the boundary. At $\alpha=0$, physically, the second-grade fluid has a faster velocity than Newtonian fluid because of the elastic properties of a second-grade fluid, which are not found in Newtonian fluids.

The influence of the Soret number is depicted in Figure 7 for the various values. It has been examined that the velocity of the fluid reduces by increasing the Soret number. The Soret effect is the ratio between temperature and concentration distribution, which causes decrease velocity of the fluid. The influence of $\mathrm{Sr}$ on the velocity shows that the magnetic parameter is stronger than the Soret number.

Figure 8 demonstrates the influence of Reynolds number Re on the velocity profile. It is noticed from the velocity profile that increasing the value of Re causes the decrease of velocity. Physically it means that Reynolds number increases the fluid density due to the fact that velocity of the fluid decreases. Reynolds number directly relates to the density; hence, by increasing $R$, it retards velocity of the fluid.

Figure 9 shows the influence of the radiation parameter $N$ on the velocity profile. It is shown in Figure 9 that the velocity of the fluid increases by increasing radiation parameter $N$, which consequently enhances thermal conductivity $k \longrightarrow \infty$, which retards the velocity of the fluid. It means that velocity reduces due to high thermal radiation.

Figures 10 and 11 depict the effect of various values of heat $\theta$ and concentration distribution $\phi$. Figure 10 shows that the greater the amount of thermal radiation $N$ is, the

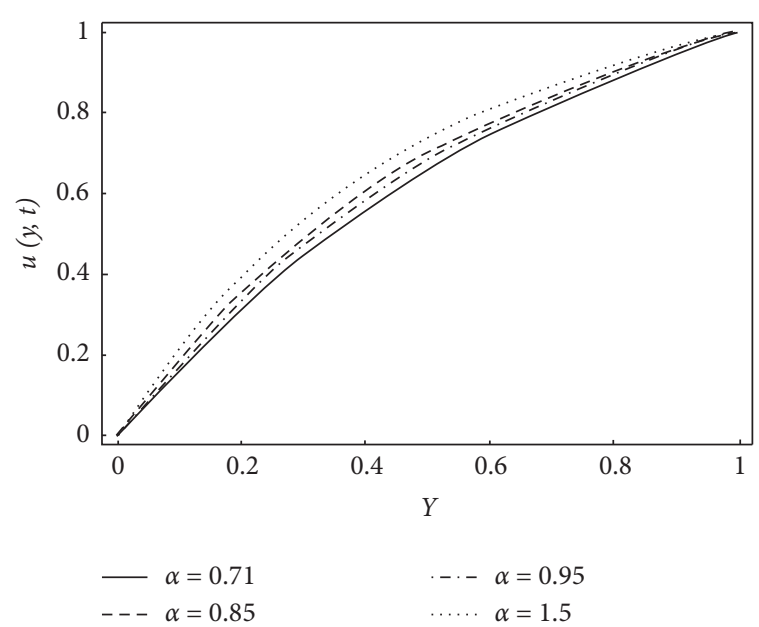

Figure 6: Velocity profile of several values for $\alpha$ when $\operatorname{Re}=5$, $\mathrm{Gr}=1.1, M=2.1, K=0.52, \mathrm{Gm}=1.1, N=0.001, \mathrm{Sr}=1.1, \varepsilon=2$, and $\omega=\pi / 2$.

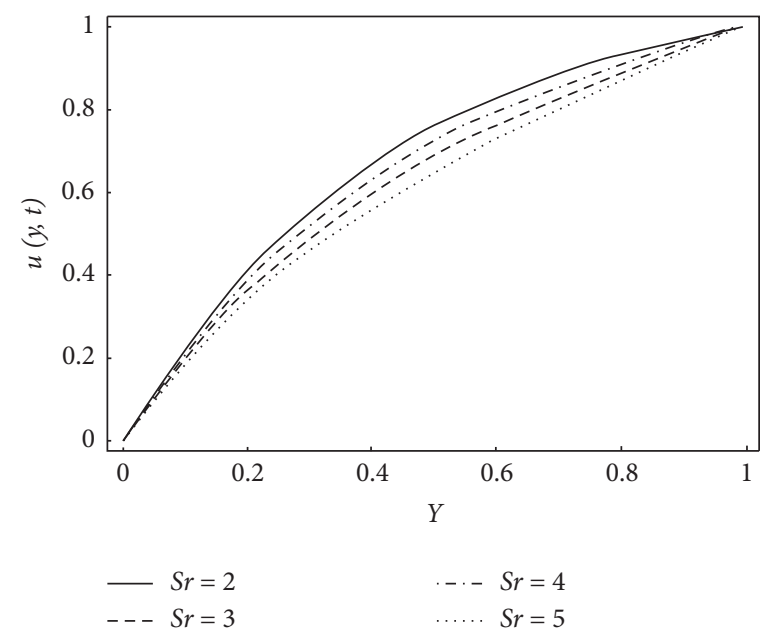

FIgURE 7: Velocity for the several values of $\mathrm{Sr}$ when $\mathrm{Re}=2.2$, $\mathrm{Gr}=2.2, M=1.1, K=1, \mathrm{Gm}=1, N=2, \alpha=7, \varepsilon=0.001$, and $\omega=\pi / 2$.

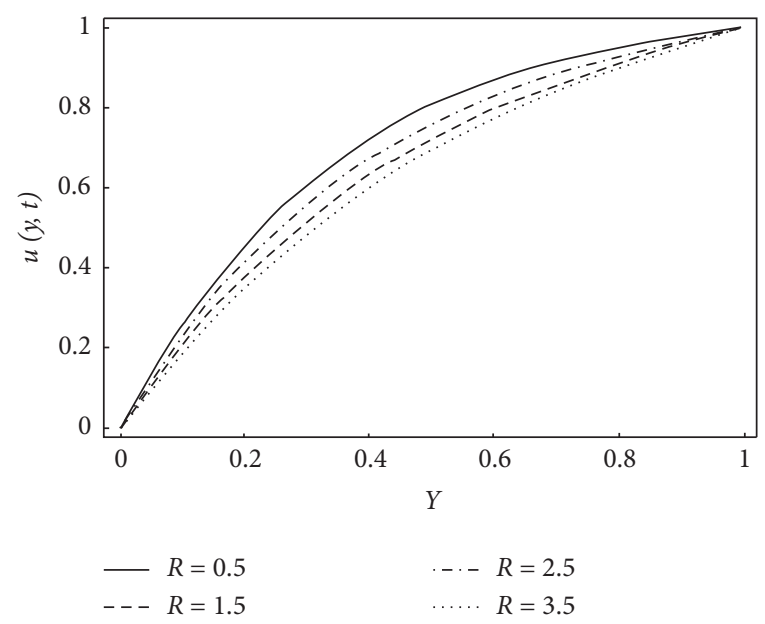

FIgURE 8: Velocity graph for the several values of Re when $\mathrm{Gr}=1.1$, $M=2.2, K=0.51, \mathrm{Gm}=1, N=2, \mathrm{Sr}=1, \alpha=0.71, \varepsilon=0.001$, and $\omega=\pi / 2$. 


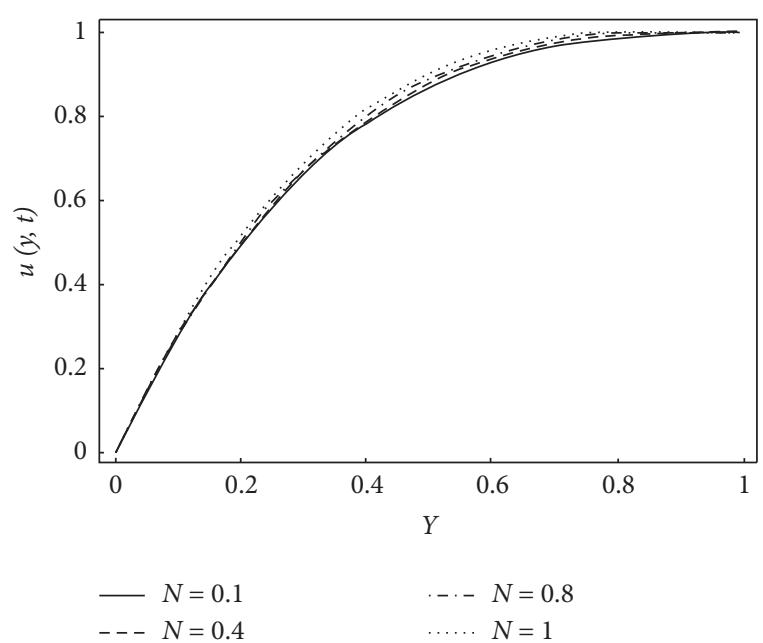

Figure 9: Velocity profile for the several values of $N$ when $\operatorname{Re}=0.2$, $\mathrm{Gr}=5, M=2, K=5, \mathrm{Gm}=0.1, \mathrm{Sr}=0.4, \alpha=0.3, \varepsilon=0.001$, and $\omega=\pi / 2$.

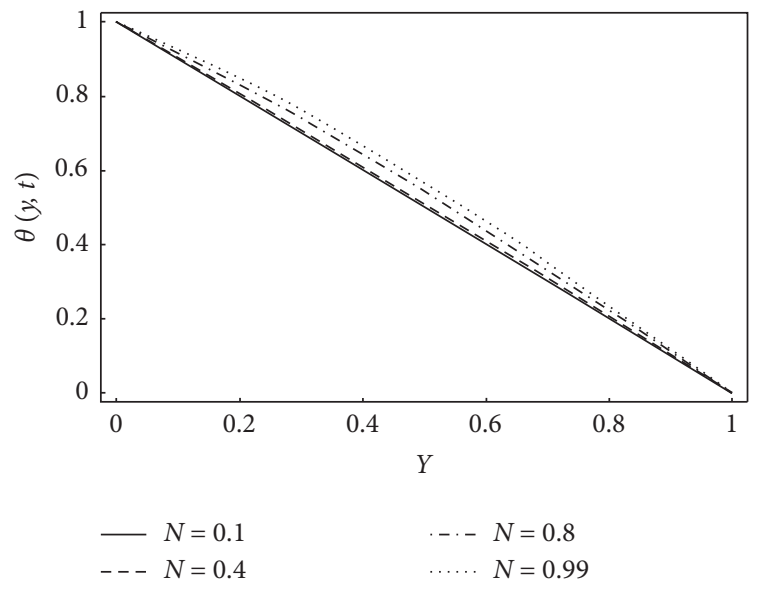

Figure 10: Effect of temperature equation for the several values of $N$ when $t=1.01$.

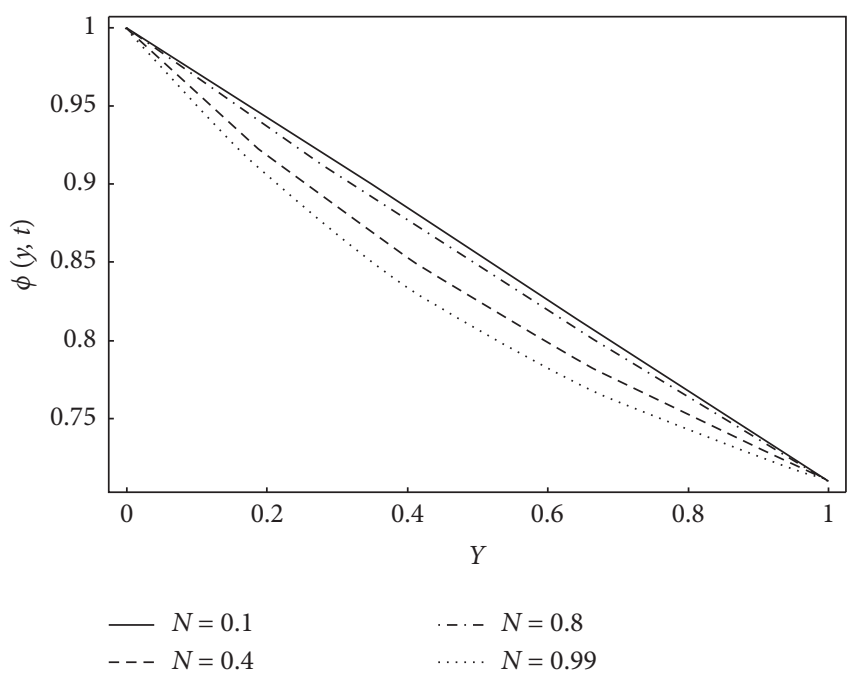

FIGURE 11: The effect of concentration equation for the various values of $N$ when $\mathrm{Sr}=0.71$.

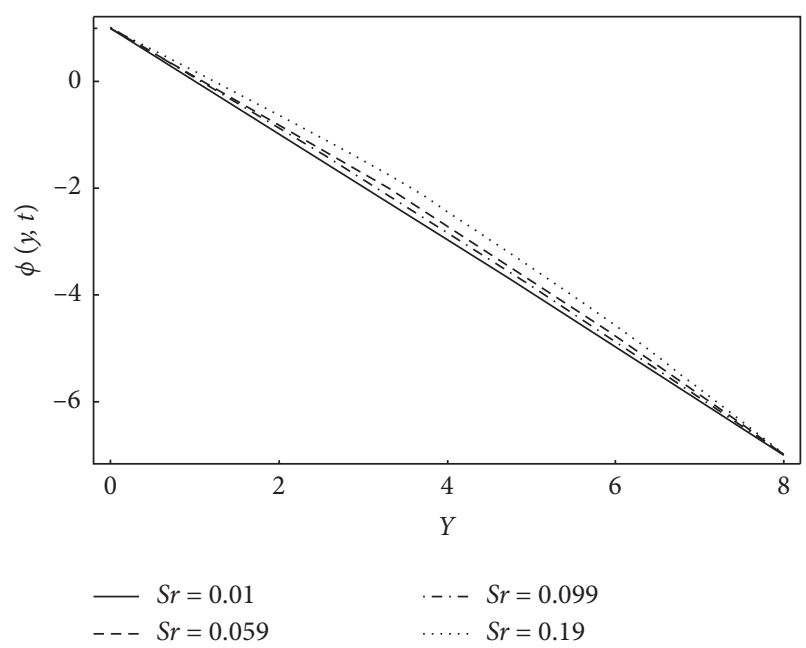

Figure 12: Effect of concentration equation for the various values of $\mathrm{Sr}$ when $N=1.1$.

more the range of the temperature rises; it means that the thermal boundary layer thickness increases with increasing $N$. Still, the concentration has opposite influence to the heat profile, which is shown in Figure 11.

Soret number's influence is shown in Figure 12, which demonstrates that it is linked to the mass transfer of the fluid flow, and Soret number is the ratio between temperature and concentration. The concentration profile shows that, by increasing Soret number, a considerable variation has been seen on the concentration profile.

The influences of several parameters are discussed in Table 1. It has been noticed that by increasing magnetic parameter $M$ that the skin friction rises. The skin friction reduces when the amount of mass Grashof number Gm rises, and permeability parameter $K$, Soret number $\mathrm{Sr}$, viscoelastic parameter, Reynolds number, and phase angle reduce skin friction, but $M$ has an opposite effect on the skin, increasing skin friction. The influences of $M$ and permeability parameters $K$ are discussed.

\section{Conclusion}

In the current work, unsteady MHD fluctuating free convection flow of second-grade fluid through a porous medium in the pressure gradient presence is studied. The effects of different parameters have been shown graphically, and skin friction is evaluated in tabular form. The perturbation technique is used to obtain exact analytical solutions for velocity, heat, and mass equations. The graphical results are discussed in detail. The primary outcomes of the present study are summarized as follows:

(1) When the viscoelastic parameter $\alpha$ and mass Grashof $\mathrm{Gm}$ increase, the fluid's velocity increases, while skin friction decreases by increasing both these parameters.

(2) Velocity and skin friction decrease for the large amounts of $\mathrm{Re}, \mathrm{Sr}$, and $K$. 
(3) When the value of magnetic parameter $M$ increased, velocity and skin friction increased.

(4) The effect of phase angle on velocity and skin friction shows that by increasing $\omega$ fluid velocity increases while the skin friction decreases.

(5) Increasing Gr the velocity of the fluid flow also increases the fluid velocity by increasing the radiation parameter $N$.

\section{Abbreviations}

$u$ : Boundary layer velocity of the fluid

$v: \quad$ The velocity of the dust particle

$U$ : Free stream velocity

$T: \quad$ The temperature of the fluid

$T_{p}$ : The temperature of the particle

$\rho: \quad$ Fluid density

$v$ : kinematic viscosity

$\alpha_{1}$ : Second-grade parameter

$K_{0}$ : Stock's resistance coefficient

$N_{0}$ : The number density of the dust particle, which is assumed to be constant

$\sigma: \quad$ Electrical conductivity

$B_{0}:$ Applied magnetic field

$g$ : Gravitational acceleration

$\beta_{T}$ : Coefficient of thermal expansion

$\beta_{C}$ : Coefficient of volumetric expansion

$k$ : Thermal conductivity of the base fluid

$c_{p}:$ Specific heat capacity

$q_{r}$ : Radiative heat flux

$\omega$ : Frequency of oscillation

$\alpha_{0}$ : Radiation absorption coefficient

Sc: Schmidt number

$\rho_{p}$ : The density of the dust particle

$\gamma_{T}$ : Temperature relaxation time

$T_{w}$ : Temperature of wall

$T_{0}$ : Ambient temperature

$c_{s}$ : Specific heat capacity of the dust particle

$m$ : The average mass of the dust particle

$M$ : Nondimensional parameter

Pe: Peclet number

Gr: Grashof number

Re: Reynolds number

$R: \quad$ Particle concentration parameter

$\phi$ : Heat absorption coefficient

$K_{1}$ : Dusty fluid parameter

$K_{2}$ : Dusty fluid parameter

$\gamma$ : Nondimensional temperature relaxation time parameter

$\alpha$ : Nondimensional second-grade parameter

$D$ : Mass diffusivity

a: Width channel

$K_{T}$ : Thermal diffusion ratio

Sr: Soret number.

\section{Data Availability}

All the data are available in the manuscript.

\section{Conflicts of Interest}

The authors declare that there are no conflicts of interest.

\section{References}

[1] R. P. Chhabra and J. F. Richardson, Non-Newtonian Flow and Applied Rheology: Engineering Applications, ButterworthHeinemann, Oxford, UK, 2011.

[2] D. O. B. Jones, A. R. Gates, V. A. I. Huvenne, A. B. Phillips, and B. J. Bett, "Autonomous marine environmental monitoring: application in decommissioned oil fields," Science of the Total Environment, vol. 668, pp. 835-853, 2019.

[3] T. C. Goede, K. G. de Bruin, and D. Bonn, "High-velocity impact of solid objects on Non-Newtonian Fluids," Scientific Reports, vol. 9, no. 1, p. 1250, 2019.

[4] T. Hayat, C. Fetecau, and M. Sajid, "On MHD transient flow of a Maxwell fluid in a porous medium and rotating frame," Physics Letters A, vol. 372, no. 10, pp. 1639-1644, 2008.

[5] I. C. Christov and C. I. Christov, "Comment on "On a class of exact solutions of the equations of motion of a second-grade fluid" by C. Fetecău and J. Zierep," ActaMechanica, vol. 215, no. 1-4, pp. 25-28, 2010.

[6] F. Ali, M. Saqib, I. Khan, N. A. Sheikh, and S. A. A. Jan, "Exact analysis of MHD flow of a Walters'-B fluid over an isothermal oscillating plate embedded in a porous medium," The European Physical Journal Plus, vol. 132, no. 2, pp. 1-14, 2017.

[7] R. J. Moreau, Magnetohydrodynamics, Springer Science \& Business Media, Berlin, Germany, 2013.

[8] H. Alfvén, "On the existence of electromagnetic-hydromagnetic waves," Arkiv for Matematik, Astronomi och Fysik, vol. 29, pp. 1-7, 1943.

[9] A. Khan, I. Khan, F. Ali, and S. Shafie, "Effects of wall shear stress on MHD conjugate flow over an inclined plate in a porous medium with ramped wall temperature," Mathematical Problems in Engineering, vol. 2014, 15 pages, 2014.

[10] R. C. Chaudhary and P. Jain, "Hall Effect on MHD mixed convection flow of a viscoelastic fluid past an infinite vertical porous plate with mass transfer and radiation," Theoretical and Applied Mechanics, vol. 33, no. 4, pp. 281-309, 2006.

[11] O. D. Makinde and P. Y. Mhone, "Heat transfer to MHD oscillatory flow in a channel filled with porous medium," Romanian Journal of Physics, vol. 50, no. 9-10, p. 931, 2005.

[12] T. Hayat, R. J. Moitsheki, and S. Abelman, "Stokes' first problem for Sisko fluid over a porous wall," Applied Mathematics and Computation, vol. 217, no. 2, pp. 622-628, 2010.

[13] F. Ali, M. Saqib, I. Khan, and N. A. Sheikh, "Application of Caputo-Fabrizio derivatives to MHD free convection flow of generalized Walters'-B fluid model," The European Physical Journal Plus, vol. 131, no. 10, pp. 1-10, 2016.

[14] O. D. Makinde and E. Osalusi, "MHD steady flow in a channel with slip at the permeable boundaries," Romanian Journal of Physics, vol. 51, no. 3-4, p. 319, 2006.

[15] O. D. Makinde and A. Aziz, "MHD mixed convection from a vertical plate embedded in a porous medium with a convective boundary condition," International Journal of Thermal Sciences, vol. 49, no. 9, pp. 1813-1820, 2010.

[16] R. N. Barik, G. C. Dash, and A. Mohanty, "Chemical reaction effect on MHD Oscillatory flow through a porous medium bounded by two vertical porous plates with the heat source and soret effect," Journal of Applied Analysis and Computation, vol. 3, no. 4, pp. 307-321, 2013.

[17] R. Sivaraj and B. R. Kumar, "Chemically reacting unsteady MHD oscillatory slip flow in a planer channel with varying 
concentration," International Journal of Mathematics and Scientific Computing, vol. 1, no. 1, pp. 35-42, 2011.

[18] K. D. Singh, "Exact solution of an oscillatory MHD flow in a channel filled with porous medium," International Journal of Applied Mechanics and Engineering, vol. 16, no. 1, pp. 277283, 2011.

[19] A. Mehmood and A. Ali, "The effect of slip condition on unsteady MHD oscillatory flow of a viscous fluid in a planer channel," Romanian Journal of Physics, vol. 52, no. 1/2, p. 85, 2007.

[20] A. Kumar, C. L. Varshney, and S. Lal, "Perturbation technique to unsteady MHD periodic flow of viscous fluid through a planer channel," Journal of Engineering and Technology Research, vol. 2, no. 4, pp. 73-81, 2010.

[21] F. Ali, I. Khan, N. Mustapha, and S. Shafie, "The unsteady magnetohydrodynamic oscillatory flow of viscoelastic fluids in a porous channel with heat and mass transfer," Journal of the Physical Society of Japan, vol. 81, no. 6, Article ID 64402, 2012.

[22] I. G. Baoku, C. Israel-Cookey, and B. I. Olajuwon, "Influence of thermal radiation on a transient MHD Couette flow through a porous medium," Journal of Applied Fluid Mechanics, vol. 5, no. 1, 2012.

[23] S. S. Motsa and S. Shateyi, "Numerical analysis of mixed convection magnetohydrodynamic heat and mass transfer past a stretching surface in a micro-polar fluid-saturated porous medium under the influence of ohmic heating," in Mass Transfer-Advances in Sustainable Energy and Environment Oriented Numerical ModelingIntechOpen, London, UK, 2013.

[24] A. A. Bakr, "Effects of chemical reaction on MHD free convection and mass transfer flow of a micropolar fluid with oscillatory plate velocity and constant heat source in a rotating frame of reference," Communications in Nonlinear Science and Numerical Simulation, vol. 16, no. 2, pp. 698-710, 2011.

[25] J. Prakash, R. Sivaraj, and B. R. Kumar, "Influence of chemical reaction on unsteady MHD mixed convective flow over a moving vertical porous plate," International Journal of Fluid Mechanics, vol. 3, no. 1, pp. 1-14, 2011.

[26] R. Kandasamy, K. Periasamy, and K. S. Prabhu, "Chemical reaction heat and mass transfer on MHD flow over a vertical stretching surface with the heat source and thermal stratification effects," International Journal of Heat and Mass Transfer, vol. 48, no. 21-22, pp. 4557-4561, 2005.

[27] A. Ishak, K. Jafar, R. Nazar, and I. Pop, "MHD stagnation point flow towards a stretching sheet," Physica A: Statistical Mechanics and Its Applications, vol. 388, no. 17, pp. 33773383, 2009.

[28] T. Akbar, R. Nawaz, M. Kamran, and A. Rasheed, "Magnetohydrodynamic (MHD) flow analysis of second grade fluids in a porous medium with prescribed vorticity," AIP Advances, vol. 5, no. 11, Article ID 117133, 2015.

[29] M. M. Rashidi, M. Ali, N. Freidoonimehr, B. Rostami, and M. A. Hossain, "Mixed convective heat transfer for MHD viscoelastic fluid flow over a porous wedge with thermal radiation," Advances in Mechanical Engineering, vol. 6, Article ID 735939, 2014.

[30] J. Jang and S. S. Lee, "Theoretical and experimental study of MHD (magnetohydrodynamic) micropump," Sensors and Actuators A: Physical, vol. 80, no. 1, pp. 84-89, 2000.

[31] M. E. Erdogan and C. E. Imrak, "On unsteady unidirectional flows of a second-grade fluid," International Journal of Nonlinear Mechanics, vol. 40, no. 10, pp. 1238-1251, 2005.
[32] C. Fetecău and J. Zierep, "On a class of exact solutions of the equations of motion of a second-grade fluid," Acta Mechanica, vol. 150, no. 1-2, pp. 135-138, 2001.

[33] M. E. Erdogan and C. E. İmrak, "On the comparison of two different solutions in the form of series of the governing equation of an unsteady flow of a second-grade fluid," International Journal of Nonlinear Mechanics, vol. 40, no. 4, pp. 545-550, 2005.

[34] M. Nazar, C. Fetecau, D. Vieru, and C. Fetecau, "New exact solutions corresponding to the second problem of Stokes for second grade fluids," Nonlinear Analysis: Real World Applications, vol. 11, no. 1, pp. 584-591, 2010.

[35] F. Ali, M. Norzieha, S. Sharidan, I. Khan, and T. Hayat, "New exact solutions of Stokes' second problem for an MHD second grade fluid in a porous space," International Journal of Nonlinear Mechanics, vol. 47, no. 5, pp. 521-525, 2012.

[36] F. Ali, N. A. Sheikh, M. Saqib, and I. Khan, "Unsteady MHD flow of second-grade fluid over an oscillating vertical plate with isothermal temperature in a porous medium with heat and mass transfer by using the Laplace transform technique," Journal of Porous Media, vol. 20, no. 8, 2017.

[37] T. Hayat, Z. Abbas, and M. Sajid, "Heat and mass transfer analysis on the flow of a second grade fluid in the presence of chemical reaction," Physics Letters A, vol. 372, no. 14, pp. 2400-2408, 2008.

[38] M. I. Anwar, S. Shafie, I. Khan, and M. Z. Salleh, "Conjugate effects of radiation flux on double-diffusive mhd free convection flow of a nanofluid over a power-law stretching sheet," ISRN Thermodynamics, vol. 2012, Article ID 217278, 7 pages, 2012.

[39] N. T. Eldabe, E. F. Elshehawey, E. M. E. Elbarbary, and N. S. Elgazery, "Chebyshev finite difference method for MHD flow of a micropolar fluid past a stretching sheet with heat transfer," Applied Mathematics and Computation, vol. 160, no. 2, pp. 437-450, 2005.

[40] E. S. Moustafa, "MHD of a fractional viscoelastic fluid in a circular tube," Mechanics Research Communications, vol. 33, no. 2, pp. 261-268, 2006.

[41] N. A. Shah and I. Khan, "Heat transfer analysis in a secondgrade fluid over an oscillating vertical plate using fractional Caputo-Fabrizio derivatives," The European Physical Journal C, vol. 76, no. 7, p. 362, 2016.

[42] P. M. Patil, D. Anilkumar, and S. Roy, "Unsteady thermal radiation mixed convection flow from a moving vertical plate in a parallel free stream: effect of Newtonian heating," International Journal of Heat and Mass Transfer, vol. 62, pp. 534-540, 2013.

[43] A. Ishak, R. Nazar, and I. Pop, "Flow and heat transfer characteristics on a moving flat plate in a parallel stream with constant surface heat flux," Heat and Mass Transfer, vol. 45, no. 5, pp. 563-567, 2009.

[44] H. S. Takhar, A. J. Chamkha, and G. Nath, "MHD flow over a moving plate in a rotating fluid with magnetic field Hall currents and free stream velocity," International Journal of Engineering Science, vol. 40, no. 13, pp. 1511-1527, 2002.

[45] K. Gharali and D. A. Johnson, "Dynamic stall simulation of a pitching airfoil under unsteady freestream velocity," Journal of Fluids and Structures, vol. 42, pp. 228-244, 2013.

[46] R. S. Alassar, H. M. Badr, and R. Allayla, "Viscous flow over a sphere with fluctuations in the free-stream velocity," Computational Mechanics, vol. 26, no. 5, pp. 409-418, 2000.

[47] S. Levy, "Effect of large temperature changes (including viscous heating) upon laminar boundary layers with variable 
free-stream velocity," Journal of the Aeronautical Sciences, vol. 21, no. 7, pp. 459-474, 1954.

[48] R. E. Kelly, "The final approach to steady viscous flow near a stagnation point following a change in free stream velocity," Journal of Fluid Mechanics, vol. 13, no. 3, pp. 449-464, 1962.

[49] R. Mei and R. J. Adrian, "Flow past a sphere with an oscillation in the free-stream velocity and unsteady drag at finite Reynolds number," Journal of Fluid Mechanics, vol. 237, pp. 323-341, 1992.

[50] N. Rott and M. L. Rosenzweig, "On the response of the laminar boundary layer to small fluctuations of the freestream velocity," Journal of the Aerospace Sciences, vol. 27, no. 10, pp. 741-747, 1960.

[51] R. Bellman, "Perturbation methods applied to nonlinear dynamics," Journal of Applied Mechanics, vol. 22, pp. 500-502, 2010. 